\title{
Evaluation of Handwritten Character with Calligraphy
}

\author{
Haruka Yamaa, Yoshiharu Koya ${ }^{*}$ \\ Kobe City College of Technology, 8-3, Gakuenhigashimachi, Nishiku, Kobe, 651-2194, Japan
}

koya@kobe-kosen.ac.jp

\begin{abstract}
In recent years, elementary schools and junior high schools have calligraphy classes in Japan, but the number of calligraphy classes is decreasing with a decreasing number of teachers. Therefore, it is difficult to improve student's calligraphy because the time allocated to learn calligraphy is not sufficient. Hence, if there is a system which automatically evaluates the character, we can improve our calligraphy by ourselves even if there is no teacher.

Many methods to learn calligraphy have been proposed before $^{(1)-(3)}$. However, these needed a special device and were difficult to use. Therefore, We have been proposed a method to learn calligraphy using an offline system. But, we just estimated the outline of the character, we did not evaluate the characters yet. In this study, we created an index to evaluate a character by finding the characteristics of a skilled hand. And, we examined an index to evaluate written characters using an offline system.
\end{abstract}

Keywords: calligraphy, character, evaluation.

\section{Introduction}

In recent years, the number of teachers of calligraphy has been decreasing so the chance for improvement in character writing decreases. Furthermore, the number of opportunities to write characters has decreased due to the spread of smartphones. Therefore it was not noticeable if we wrote beautiful characters or not.

In addition, there are two problems when foreigners and children write Japanese words. One problem is the balance of character, the other problem is stroke order. If we can solve these problems, we can improve calligraphy by ourselves even if there is no teacher.
Therefore at first we suggested a method to extract the outline of the character to get the form of the character ${ }^{(4)}$. Using the pixel information of the character, we can get various indexes even if we do not use an outline when we analyze the character. But, the result differs due to the difference in the number of strokes. Therefore we extracted an outline and got characteristics. In this study, we compared a character with the character of a skilled hand, and we got an index for improvement to evaluate.

\section{Extraction of character outline}

\subsection{Extraction of candidate points of outline}

We show a sample image of candidate points in figure 1. The red points are candidate points which are the farthest black pixels from the center point. In this study, we defined the center of the character as center point. The outline in this study is formed by Fourier series to a predetermined second order which is obtained by expanding the candidate points to a Fourier series based on an ellipse.

\subsection{Extraction of outline $e^{(5)}$}

In figure 2, $M$ represents a proper subspace of Hilbert space $H$, and is consisted of an ideally band-limited signal $x(t) . N$ is observation space that contains $y(t) . y(t)$ is an observation signal partially lost an amplitude information of $x(t)$. Missing operator

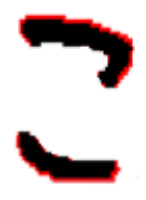

Fig. 1. Sample image of candidate points 


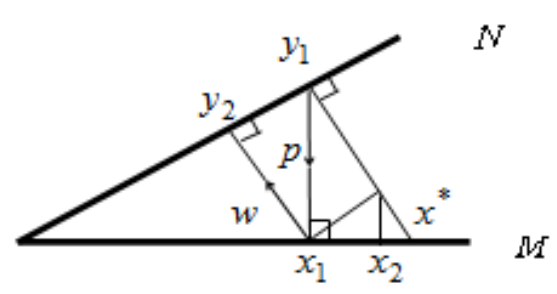

Fig. 2. Principle of extraction of contour

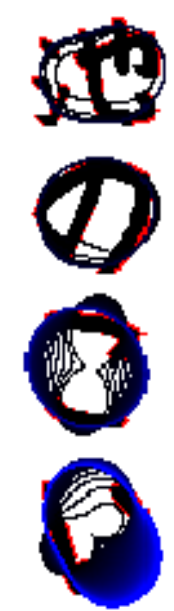

(a) Iterative result

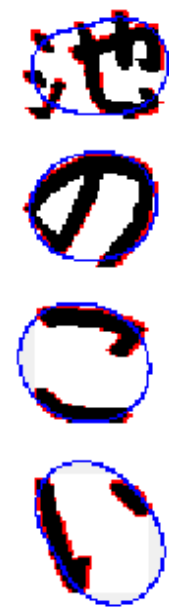

(b)Final result
Fig. 3. Extraction of contour

$w(t)$ mapped $x(t)$ to $y(t)$. Band-limiting operator $p(t)$ mapped $y(t)$ to $x(t) . p(t)$ is also a projection operator. Operator $w(t)$ and $p(t)$ are realized using a window function and DFT, respectively.

\subsection{Necessity of outline}

Some people think that it is not necessary to use an outline. Therefore we will explain the need of the outline. We show centroid position of horizontal axis in each character in table 1.

Original in table 1 is centroid position as center of character. That is a center from left side to right side of character. Outline in table 1 means a centroid was calculated using outline only. Black pixel in table 1 means a centroid is calculated using black pixel only. Therefore, if the length of the stroke between left and right is different, like the fourth character, the centroid position is changed. However, we could reduce these influence when we use an outline. This result affects many indexes as well as a centroid position. Therefore we evaluated the character using outline although the amount of calculation increased.
Table 1. Centroid position(unit:pixel).

\begin{tabular}{|c|c|c|c|}
\hline Character & Original & Outline & Black pixel \\
\hline 1st & 29 & 30 & 32 \\
\hline 2nd & 28 & 28 & 29 \\
\hline 3rd & 28 & 27 & 27 \\
\hline 4th & 29 & 30 & 23 \\
\hline
\end{tabular}

\section{Index of evaluation}

The purpose of this study is to evaluate the character and give beginner writers advice for improvement. Therefore, we must decide an index for evaluating a written character. The index which we used follows.

(a) The size of character

(b) The ratio of character length

(c) Inclination

(d) Width of the stroke

(e) The position of centroid

(f) Stroke order

\subsection{Calculation of eigenvalue and eigenvector}

We calculated eigenvalue and eigenvector for calculation of the character size, character length ratio and inclination. Because a big eigenvalue is calculated so that a big character, can use the eigenvalue for an index of the evaluations. In addition, the form of outline is calculated by eigenvalue. If the character has good balance, the form of the outline becomes a circle. Furthermore, we can get the inclination of the character from eigenvector. We used equation(1) and equation(2) for calculation of eigenvalue and eigenvector.

The equation $f(\theta)$ in equation(2) is an outline which is an approximation by Fourier series. $\lambda$ is eigenvalue and $\mathbf{u}$ is eigenvector. We show the result that we calculated using these equations in table 2.

$$
\mathbf{A}=\left[\begin{array}{cc}
\sum^{\mathbf{A u}}=\boldsymbol{\lambda \mathbf { u }} \\
\sum_{\theta=0}^{359}\{\mathrm{f}(\theta) \cos \theta\}^{2} & \sum_{\theta=0}^{359}\{\mathrm{f}(\theta) \cos \theta\}\{\mathrm{f}(\theta) \sin \theta\} \\
\sum_{\theta=0}^{359}\{\mathrm{f}(\theta) \cos \theta\}\{\mathrm{f}(\theta) \sin \theta\} & \sum_{\theta=0}^{359}\{\mathrm{f}(\theta) \sin \theta\}^{2}
\end{array}\right]
$$

We understood that a eigenvalue becomes big if a character was big from table 2. Because black pixels (character domain) spread, a eigenvalue becomes big,. Furthermore, it is difficult to fix the balance of a character 
Table 2. Eigenvalue and Eigenvector.

\begin{tabular}{|c|c|c|c|}
\hline Character & $\begin{array}{c}\text { Max } \\
\text { eigenvalue }\end{array}$ & $\begin{array}{c}\text { Ratio of } \\
\text { eigenvalue }\end{array}$ & $\begin{array}{c}\text { Inclination } \\
\text { (degree) }\end{array}$ \\
\hline 1st & 23 & 1.15 & 4.0 \\
\hline 2nd & 23 & 1.10 & -12.1 \\
\hline 3rd & 24 & 1.04 & 25.8 \\
\hline 4th & 26 & 1.24 & -35.3 \\
\hline
\end{tabular}

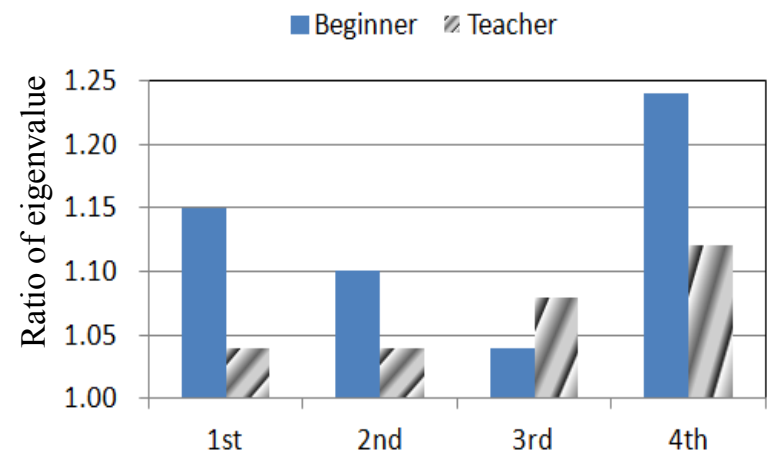

Characters

Fig. 4. Ratio of eigenvalue

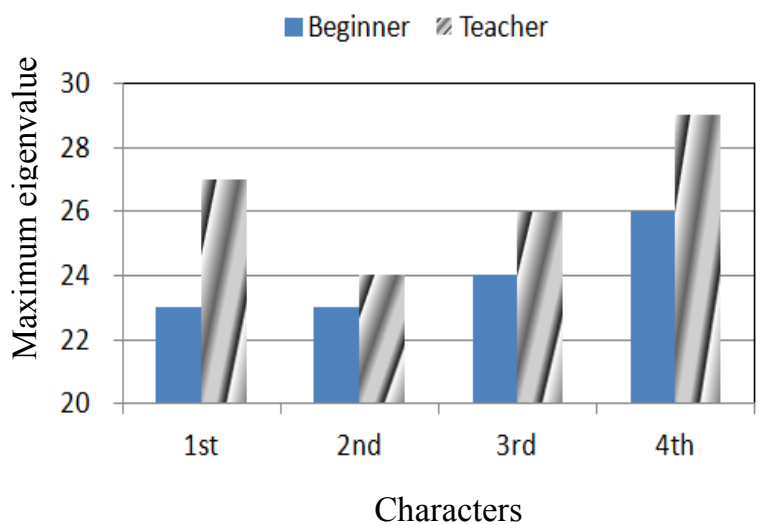

Fig. 5. Maximum eigenvalue

with fewer strokes, and the form of character is like an oval. And, we show the result of ratio of eigenvalue and maximum eigenvalue of comparison between beginner and teacher in figure 4 and figure 5 . We could not get an index of inclination because the inclination of characters are different for each character.

\subsection{Thinning of character}

Next, we think about the width of the stroke. About the width, we use a method to thin a stroke, and count black pixels in a normal direction. For example, the Hilditch thinning algorithm is a method to make it thinner. We show thinning images in figures 6(b) and figure7(b).

The calligraphy has an important element which is the width of the stroke. Particularly, the stopping part has a

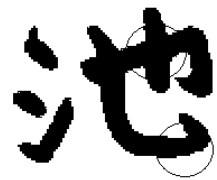

(a) original image

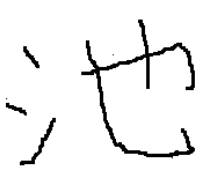

(b)thinning image
Fig. 6. Thinning result of beginner

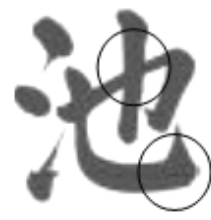

(a) original image

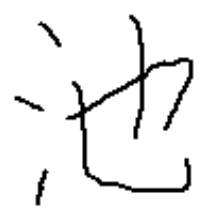

(b)thinning image
Fig. 7. Thinning result of teacher

Table 3. Width of stroke

\begin{tabular}{|c|c|}
\hline stroke & $\begin{array}{c}\text { Width } \\
\text { (pixel) }\end{array}$ \\
\hline Upper domain & 8 \\
\hline Lower domain & 6 \\
\hline
\end{tabular}

Table 4. Width of stroke

\begin{tabular}{|c|c|}
\hline stroke & $\begin{array}{c}\text { Width } \\
\text { (pixel) }\end{array}$ \\
\hline Upper domain & 4 \\
\hline Lower domain & 9 \\
\hline
\end{tabular}

wide, and then narrow part if a Chinese brush is moved smoothly. Therefore, we can get information of how to use a Chinese brush by extracting this element. We show the result in figure6 and figure7. Please pay attention to a circle in figure 6(a) and figure7(a). Because the Chinese brush was moved smoothly with a narrow stroke, domain of wide width happens once Chinese brush stops. Therefore, we counted the number of black pixels of the normal direction from the thinning image. We show the result in table 3 and table4. But, even using the same stroke, the ratio of width is different, and thickness is opposite. So, we can get the strength and weakness of the power applied to the Chinese brush as a numerical value.

\subsection{Position of centroid}

We showed the position of centroid in table 1 . In this case, centroid were almost the same if we did not use the centroid by black pixel. 


\section{Conclusion}

In recent years many foreigners came to Japan, and opportunities to learn Japanese increased. In addition, the number of people who practice Japanese and who want to improve writing Japanese increased. Therefore, in this study, we propose a system which evaluates a character with an offline system. If it is online, we can get different stroke orders. But, in this study, as the system is offline, it is difficult to get stroke orders. So, this problem is for future work in this study. However we were able to make a system which helps students learn using five indexes. We will consider giving you advice from the result of these indexes in the future.

\section{References}

(1) Motoki Onuma, Masaki Nakagawa : "An On-line Writing-box-free Line-direction Free and Character-orientation Free Recognition System for Handwritten Text ", The Institute of Electronics, Technical Report of IEICE, Information and Communication Engineers, 102, 555, pp.49-55 (2003-01)(in Japanese)

(2) Masayoshi Okamoto, Kazuhiko Yamamoto : “On-line Handwriting Character Recognition using Directional Features and Direction-Change Features", T.IEEJ Japan, Vol.119-C No.3 pp.358-366 (1999-3) (in Japanese)

(3) Hideyuki Shimada, Yasuhiro Shimada, Mitsuru Okura : "A Virtual Calligraphy System Drawable with a Chinese Brush", Information Processing Society of Japan, Vol.47, No.12,pp.3392-3401(2006) (in Japanese)

(4) Haruka Yamaa, Yoshiharu Koya : " A Study of Offline Calligraphy Learning System", Extended Abstract of the 49th ISCIE International Symposium on Stochastic Systems Theory and Its Applications, pp.31-32 (2017)

(5) Yoshiharu Koya, Masashige Hori, Isao Mizoshiri:'Border Detection of Echocardiogram using Projection Operator", T.IEEJ Japan, Vol.120-C No.8/9 pp.1236-1241 (2000-8) (in Japanese) 